\title{
Immediate efficacy of percutaneous transhepatic obliteration and sclerotherapy for giant pipeline esophageal varices hemorrhage in a patient with liver cirrhosis type $\mathrm{C}$
}

Toru Ishikawa $^{1 *}$, Michitaka Imai ${ }^{1}$, Takashi Owaki ${ }^{1}$, Hiroki Sato ${ }^{1}$, Yujiro Nozawa ${ }^{1}$, Tomoe Sano ${ }^{1}$, Akito Iwanaga ${ }^{1}$, Keiichi Seki ${ }^{1}$, Terasu Honma $^{1}$ and Toshiaki Yoshida ${ }^{1}$

${ }^{1}$ Department of Gastroenterology and Hepatology, Saiseikai Niigata Daini Hospital, Niigata, Japan

A 49-year-old man was referred to us with a diagnosis of hematemesis by giant pipeline esophageal varices due to liver cirrhosis hepatitis $\mathrm{C}$ infection after carving a tattoo at 20-year age (Figures $1 \mathrm{~A}$ \& 1B). We reviewed the treatment strategy. Endoscopic injection sclerotherapy (EIS) was generally treated esophageal varices. However, we diagnosed to need a large amount of sclerosing agent, namely $5 \%$ ethanolamine oleate iopamidol (Oldamin; Mochida Pharmaceutical, Tokyo, Japan) for successful treatment. So, we decided to perform percutaneous transhepatic obliteration (PTO) and sclerotherapy (PTS) safely. Percutaneous transhepatic puncture of the intrahepatic branch of the portal vein was performed using an 18-gauge needle under sonographic guidance. A 5-French gauge sheath catheter was then introduced into the portal vein. Direct portography was performed to identify the feeding and draining veins from the left gastric vein to the collateral veins including paraesophageal vein, which caused esophageal varices hemorrhage (Figure 2A). A coaxial catheter was inserted into these feeding veins while avoiding the main feeding vein. The feeding veins were embolized with microcoils or a sclerosing agent, namely $5 \%$ ethanolamine oleate iopamidol (Oldamin; Mochida Pharmaceutical, Tokyo, Japan). PTO is usually performed by placing metallic coils in the afferent veins to reduce blood flow. Hence, PTS is usually performed by injection of sclerosing agent in the afferent veins to reduce blood flow [1] (Figure 2B). Percutaneous transhepatic portography after treatment showing that the varix and its feeder were embolized (Figure 2C). One day after PTO/PTS, giant pipeline esophageal varices disappeared (Figure 1C).

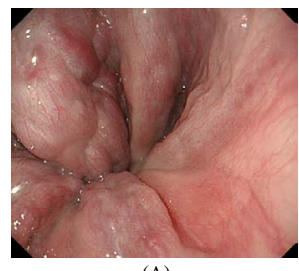

(A)

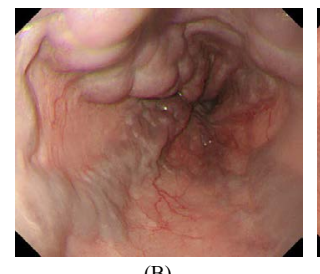

(B)

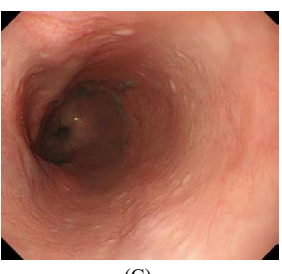

(C)
Figure 1. (A)(B)Esophagogastroduodenoscopic findings revealed giant pipeline esophageal varices from lower to upper esophagus.

(C) One day after PTO/PTS, giant pipeline esophageal varices disappeared.
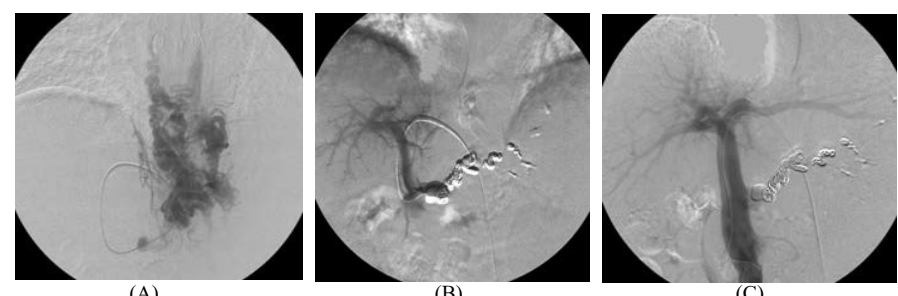

Figure 2. (A) Percutaneous transhepatic portography shows from the left gastric vein to the collateral veins including paraesophageal vein, which caused esophageal varices hemorrhage.

(B) The feeding veins were embolized with microcoils and a sclerosing agent.

(C) Percutaneous transhepatic portography after treatment showing that the varix and its feeder were embolized.

PTO was firstly reported by Lunderquist in 1974 [2]. This treatment had been widely performed as an emergency measure in cases of variceal hemorrhage [2]. Moreover, PTS is usually performed by placing metallic coils in the afferent veins to reduce blood flow into the gastric varix, after which a sclerosing agent is injected in the antegrade direction into the gastric varix [1]. When there are multiple afferent veins, this procedure may need to be performed for all these veins. Percutaneous transhepatic obliteration (PTO) and percutaneous transhepatic sclerotherapy (PTS) are widely performed as an emergency measure in cases of variceal hemorrhage. Furthermore, hepatic functional reserve is improved in this case because portal flow improved in the antegrade direction [3].

\section{References}

1. Ninoi T, Nakamura K, Kaminou T, Nishida N, Sakai Y, et al. (2004) TIPS versus transcatheter sclerotherapy for gastric varices. AJR Am J Roentgenol183: 369-376. [Crossref]

2. Lunderquist A, Vang J (1974) Transhepatic catheterization and obliteration of the coronary vein in patients with portal hypertension and esophageal varices. $N$ Engl J Med291: 646-649.[Crossref]

3. Ishikawa T, Imai M, Ko M, Sato H, Nozawa Y, et al. (2017) Percutaneous transhepatic obliteration and percutaneous transhepatic sclerotherapy for intractable hepatic encephalopathy and gastric varices improves the hepatic function reserve. Biomed Rep 6: 99-102.[Crossref]

Correspondence to: Toru Ishikawa, M.D., Department of Gastroenterology and Hepatology, Saiseikai Niigata Daini Hospital, Teraji 280-7, Niigata 950-1104, Japan, Tel: 81-25-233-6161, Fax: 81-25-233-8880,E-mail: toruishi@ngt.saiseikai. or.jp

Received: May 08, 2017; Accepted: May 19, 2017; Published: May 22, 2017
Copyright: (C2017 Yanamandra K. This is an open-access article distributed under the terms of the Creative Commons Attribution License, which permits unrestricted use, distribution, and reproduction in any medium, provided the original author and source are credited. 